\title{
A Canine Case of Partial Heterotaxia Detected by Radiography and Ultrasound
}

\author{
Hideki KAYANUMA, Tsunenori SUGANUMA, Takuo SHIDA and Shinobu SATO1) \\ Department of Veterinary Radiology, College of Veterinary Medicine, Azabu University, 1-17-71 Fuchinobe, Sagamihara, Kanagawa, \\ 229-8501 and ${ }^{1}$ Sato Veterinary Hospital, 3-1-7 Minaminaruse, Machida, Tokyo 194-0045, Japan
}

(Received 14 September 1999/Accepted 3 April 2000)

ABSTRACT. With radiography and ultrasound, reversed positioning of the fundus ventriculi and pylorus, a duodenum running on the left side, transposition of the kidneys, and normal thoracic organs were found in a 5-month-old miniature dachshund that presented with anorexia and weight loss. The case was diagnosed as partial heterotaxia. Gross observation revealed partial heterotaxia, polysplenia, abnormal lobulation of the liver, and absence of the greater omentum. These findings were consistent with those observed in aspleniapolysplenia syndrome in humans.

KEY WORDS: canine, heterotaxia, polysplenia.

J. Vet. Med. Sci. 62(8): 897-899, 2000

Heterotaxia is classified into 2 types, which are complete heterotaxia, characterized by transposition of all organs in the body cavity, and partial heterotaxia, characterized by transposition of some organs in the body cavity [1]. Since partial heterotaxia is frequently associated with asplenia or polysplenia in humans, it is called asplenia or asplenia-polysplenia syndrome [1]. Diagnosis of this syndrome depends on assessing anatomical abnormalities detected using various imaging [9, 12]. This report concerns laboratory and pathological findings in a dog with partial heterotaxia accompanied by polysplenia, abnormal hepatic lobulation, a patent ductus venosus, and absence of the greater omentum.

A 5-month-old female miniature dachshund, weighing 1.7 $\mathrm{kg}$, was brought to the Veterinary Teaching Hospital of Azabu University with abnormal behavior, occasional seizures, anorexia, and emaciation. At admission, the dog was thin and underdeveloped. On laboratory examination, abnormal findings included a slight decrease in total protein (TP, $5.8 \mathrm{~g} / \mathrm{d} l$ ) and marked elevations of alanine aminotransferase (237 U/l), alkaline phosphatase $(846 \mathrm{U} / l), \mathrm{NH}_{3}(809 \mu \mathrm{mol} / l)$, and fasting total serum bile acids (TSBA, $25 \mu \mathrm{mol} / l$ ). In addition, a significant increase in TSBA $(200 \mu \mathrm{mol} / l)$ was observed $2 \mathrm{hr}$ after feeding. Transposition of the stomach and duodenum, normal positioning of the large intestine, a shrunken liver, and no abnormalities of thoracic organs were demonstrated by radiography, including a contrast study (Fig. 1). Ultrasonography showed hypoplastic portal veins and a gallbladder that appeared normal except for its position, which was a little to the left of normal. Further, the right kidney was found to be located caudally from normal (at the 13th rib), and the left kidney was located cranially from normal (at the 12th intercostal space), although no morphological changes were observed in both kidneys. A hypoplastic spleen was observed at the right 13th rib. These findings suggested the possibility of the presence of a portosystemic shunt and transposition of some visceral organs.

An exploratory laparotomy was performed under general anesthesia to look for a portosystemic shunt. Portography revealed a single intrahepatic shunt, which could not be ligated, despite our every possible attempt. On postoperative day 3 , the dog died suddenly. A necropsy was performed.

No abnormalities were detected in the heart, lungs, and vascular branches in the thorax. In the abdomen, the fundus ventriculi was seen on the right side, and the pylorus, duodenum, and pancreas were on the left side. However, the cecum and colon were located normally. There were two spleens: one was located on the right side, as occurs in gastroduodenal situs inversus, and the other was located on the left, caudal to the pylorus. No greater omentum was found (Fig. 2). The left and right kidneys were situated toward the middle of the 12th thoracic vertebra and the middle of the first lumbar vertebra,

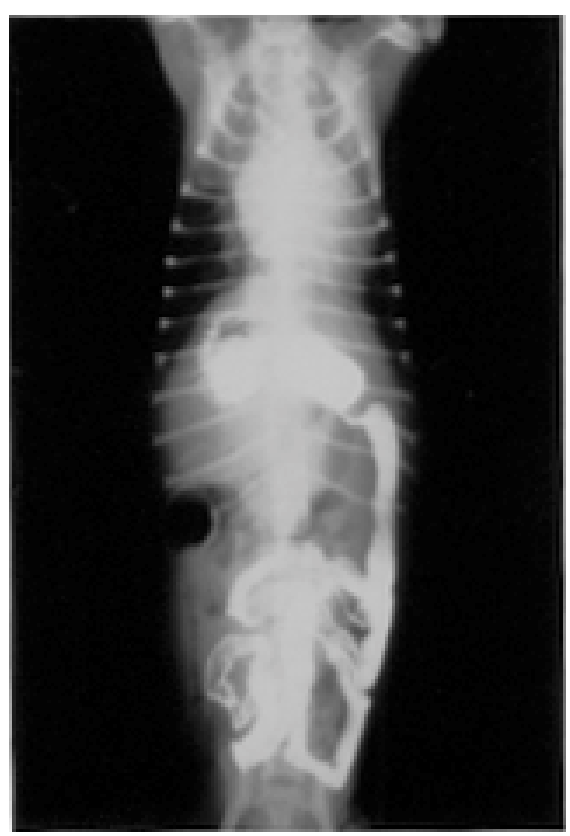

Fig. 1. Ventrodorsal view of the barium study on the radiographic examination. The fundus was located at right side. The pylorus and duodenum were recognized at the left. 


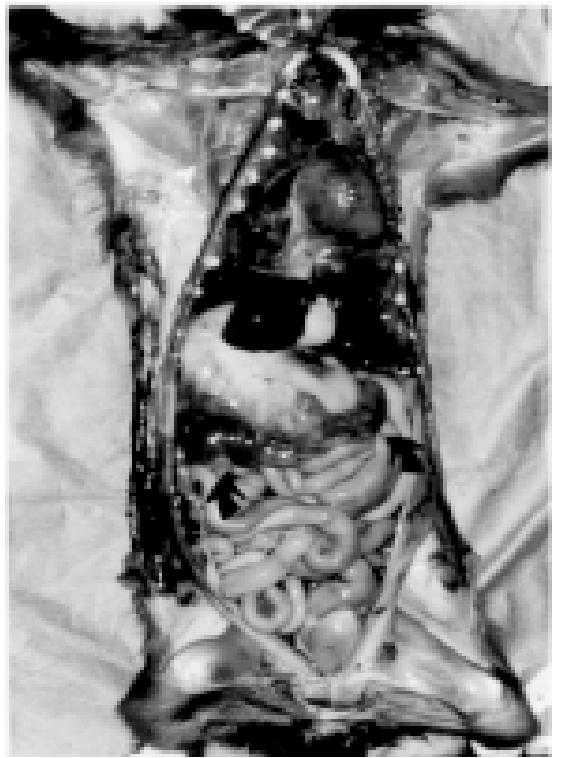

Fig. 2. Gross appearances in the dog. The lungs and heart are normal, whereas the stomach is in the reversed position. Two lobes of the spleen (arrows) are seen caudal to the stomach. The greater omentum is absent.

respectively. The liver was hypoplastic and showed abnormal symmetric lobulation, with two lobes on each of the right and left sides. The gallbladder lay between right and left medial lobes (Fig. 3).

In humans, splenic malformations include asplenia, splenic hypogenesis, spleen accessories, and polysplenia. Asplenia, splenic hypogenesis, and polysplenia are frequently complicated by cardiac and vascular abnormalities and/or partial heterotaxia $[1,2,6]$. Splenic malformation complicated by cardiac/large vessel malformation and/or heterotaxia is generally called "asplenia syndrome" or "asplenia-polysplenia syndrome" $[1,11]$. In a study of 78 asplenic humans, cardiac abnormalities and heterotaxia, cardiac abnormalities, and asplenia alone were detected in $37.2 \%, 25.6 \%, 7.7 \%$, and $5.1 \%$, respectively [8]. It has been reported that $40 \%$ of asplenia and polysplenic cases have digestiv tract disorders [6]. In another study of 85 humans with asplenia, 45 were determined to have malformations in the systemic venous system [4]. In these reports, partial heterotaxia was highly related to asplenia or polysplenia, though complete heterotaxia was very rarely associated with either asplenia or polysplenia [1]. A tubular stomach, absence of the greater omentum, malrotation of the bowel, an abnormal lobulation of the lung and liver, such as bilateral symmetry, have also been reported as malformations associated with asplenia or polysplenia $[1,2,7]$. In a report on asplenia-polysplenia syndrome, Moller et al. stated that splenic agenesis associated with symmetric lobulation of the lung and liver results from predominant right-sidedness and an isomeric left side, whereas predominant left-sidedness and an

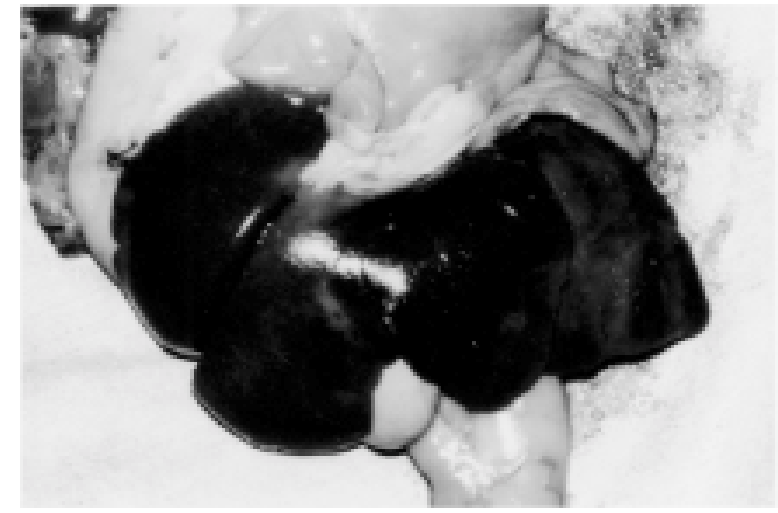

Fig. 3. Photograph of the liver and gallbladder of the dog (diaphragmatic aspect). There is a hypoplastic liver. The liver appears to be bilateral symmetric lobration. The gallbladder is in the center of right and left medial lobes.

isomeric right side results in polysplenia associated with symmetric lobulation of the lung and liver [5]. Although there are no available data on these malformations in dogs, anatomical features reported in human asplenia-polysplenia syndrome are similar to those of the case of partial heterotaxia reported here, especially symmetric hepatic lobulation and two spleens. In this case, morphological features of the liver suggested defects of both caudate and quadrate lobes, but actual defective lobes could not be identified because the marked morphological differences of the liver from normal interfered with identification in this case. The hepatic hypoplasia was thought to be caused by the portosystemic shunt. Based on recent reports on the expression of the homeobox gene Pitx2, which controls leftright asymmetry through promotion of embryonic looping of linear hearts and guts of mice, frogs, and zebrafish [3, 10], we hypothesized that this gene involves the occurrence of partial heterotaxia, like the case described here, resulting in abnormal looping of the involved organs. Hence, more detailed investigation on the etiology of asplenia/polysplenia and heterotaxia, especially its genetic factor induced by Pitx2, is required in animals.

\section{REFERENCES}

1. Aeba, S. 1974. Hukubu no kikei. p. 249, pp. 271-274. In: Gendai Gekagaku Taikei (Kimoto, S. ed.), Nakayma Syoten, Tokyo (in Japanese).

2. Bowers, P. N., Brueckner, M. and Yost, H. J. 1996. Semin. Perinatol. 20: 577-588.

3. Campione, M., Steinbeisser, H. and Schweickert, A. 1999. Development 126: 1225-1234.

4. Gilbert, E. F., Nisimura, K. and Wedum, B. G. 1958. Circulation 17: 72-86.

5. Moller, J. H., Nakib, A., Anderson, R. C. and Edwards, J. E. 1967. Circulation 36: 789-799.

6. Nakada, K., Kawaguchi, F., Wakisaka, M., Nakada, M., Enami, T. and Yamato, N. 1997. J. Pediatr. Surg. 32: 91-94.

7. Norman, A. P. 1963. Congenital Abnormalities in Infancy, Blackwell Scientific Publications, Oxford. 
8. Putchar, W. G. J. and Manion, W. C. 1956. Am. J. Clin. Pathol. 26: 429-470.

9. Sasaki, S., Matuo, Y., Takahashi, S. and Kishikawa, T. 1992. Nippon Igaku Hoshasen Gakkai Zasshi 52: 1620-1625.

10. Tsang, T. E., Kinder, S. J. and Tam, P. P. 1999. Cell. Mol. Biol.
(Noisy-Le-Grand) 45: 493-503.

11. Warkany, J. 1971. Congenital Malformations, Year Book Medical Publishers, Chicago.

12. Winer-Muram, H. T., Tonkin, I. L. and Gold, R. E. 1991. J. Thorac. Imaging 6: 69-71. 